\title{
Investigation of the integral manifolds of singularly perturbed functional differential equations
}

Nikolai Perestyuk and Ihor M. Cherevko 


\title{
INVESTIGATION OF THE INTEGRAL MANIFOLDS OF SINGULARLY PERTURBED FUNCTIONAL DIFFERENTIAL EQUATIONS
}

\author{
Nikolai A. Perestyuk and Ihor M. Cherevko \\ Mechanical and Mathematical Faculty, Kyiv National Taras Shevchenko University \\ Volodymyrska, 64, 01033, Kyiv, Ukraine \\ pmo@mechmath.univ.kiev.ua, cherevko@math.chnu.cv.ua
}

[Received: December 14, 2001]

\begin{abstract}
A system of nonlinear singularly perturbed functional differential equations with time lag in fast and slow variables is considered. The conditions of existence of the integral manifolds are established, application of the method of integral manifolds in stability problem are analyzed.
\end{abstract}

Mathematical Subject Classification: 34C45, 34D15, 34K20

Keywords: integral manifold, singular perturbation, functional differential equation

\section{Preliminaries}

A method of integral manifolds is an effective algorithm to study singularly perturbed ordinary differential equations $[1-3]$, systems with time lag $[4,5]$ and functional differential equations [6-8]. The case of systems with time lag only in fast variables is discussed in $[4,5,7,8]$ and only in slow variables in $[6,9]$. In this paper we consider a singularly perturbed system of functional differential equations with time lag both in fast and in slow variables. For such systems we establish the existence conditions of stable, and center-stable, center, center-unstable integral manifolds and consider their application for the investigation of solution stability. For ordinary differential equations analogous problems were studied in $[3,10]$.

Let $R^{n}$ be $n$-dimensional Euclidean space, $C_{\Delta}=C[-\Delta, 0], C_{\varepsilon \Delta}=C[-\varepsilon \Delta, 0]$ are the spaces of $[-\Delta, 0],[-\varepsilon \Delta, 0]$ continuous $n$-dimensioned functions.

Consider the system of functional differential equations

$$
\begin{gathered}
\frac{d x}{d t}=A x_{t}+f\left(t, x_{t}, y_{t}, \varepsilon\right), \\
\varepsilon \frac{d y}{d t}=B(t) y_{t}+g\left(t, x_{t}, y_{t}, \varepsilon\right),
\end{gathered}
$$

where $x \in R^{n}, y \in R^{m}, x_{t}=x(t+\theta), y_{t}=y(t+\varepsilon \theta),-\Delta \leq \theta \leq 0, \Delta>0, \varepsilon>0$ is a small parameter. Functions $f(t, x, y, \varepsilon), g(t, x, y, \varepsilon)$ are defined in domain $\{t \in R, x \in$ 
$\left.C_{\Delta}, y \in C_{\varepsilon \Delta}, \varepsilon \in\left[0, \varepsilon_{0}\right]\right\}$ with values in $R^{n}$ and $R^{m} . A, B$ are linear operators, given by

$$
A \varphi=\int_{-\Delta}^{0}\left[d \eta_{1}(\theta)\right] \varphi(\theta), \quad B(t) \varphi=\int_{-\Delta}^{0}\left[d \eta_{2}(t, \theta)\right] \varphi(\varepsilon \theta),
$$

where $\eta_{1}(\theta)$ is $n \times n$ matrix, whose elements are functions of limited variation; $\eta_{2}(t, \theta)$ is $m \times m$ matrix, whose elements are functions of limited variation on $\theta$ for all $t$ and uniformly continuous on $t$ with respect to $\theta$ and bounded for $t \in R$.

Suppose that for system (1.1) the following conditions are valid:

I) all roots of the characteristic equation

$$
\operatorname{det}\left(\lambda E-\int_{-\Delta}^{0}\left[d \eta_{2}(t, \theta)\right] e^{\lambda \theta}\right)=0
$$

are located in the left half-plane $\operatorname{Re} \lambda<-2 \mu<0$;

II) functions $f, g$ are continuous, bounded by constant $K$ and satisfy the following inequalities:

$$
\begin{aligned}
|f(t, x, y, \varepsilon)-f(t, \bar{x}, \bar{y}, \varepsilon)| & \leq L(\varepsilon)(|x-\bar{x}|+|y-\bar{y}|), \\
|g(t, x, y, \varepsilon)-g(t, \bar{x}, \bar{y}, \varepsilon)| & \leq L(\varepsilon)(|x-\bar{x}|+|y-\bar{y}|),
\end{aligned}
$$

where $L(\varepsilon) \rightarrow 0$ if $\varepsilon \rightarrow 0$.

\section{System transformation}

Consider the linear autonomous functional differential equation

$$
\frac{d x}{d t}=A x_{t}
$$

and its charateristc quasipolynomial

$$
H(\lambda)=\operatorname{det}\left(\lambda E-\int_{-\Delta}^{0}\left[d \eta_{1}(\theta)\right] e^{\lambda \theta}\right) .
$$

Define the shift operator, corresponding to equation (2.1), by the relation $T(t) \varphi=$ $x_{t}(\varphi)$, where $x_{t}(\varphi)$ is the solution of (2.1) with initial value $\varphi \in C_{\Delta}$ at $t=0$.

Let us introduce the following notation:

$$
\begin{gathered}
\Lambda_{1}=\{\lambda: H(\lambda)=0, \operatorname{Re} \lambda>0\}, \quad \Lambda_{2}=\{\lambda: H(\lambda)=0, \operatorname{Re} \lambda=0\}, \\
\Lambda_{3}=\{\lambda: H(\lambda)=0, \operatorname{Re} \lambda<0\} .
\end{gathered}
$$

It is known [11] that there is only a finite number of roots of equation $H(\lambda)=0$ in any half-plane $\operatorname{Re} \lambda \geq \gamma$, so the sets $\Lambda_{1}, \Lambda_{2}$ are finite dimensional.

The sets $\Lambda_{i}, i=\overline{1,3}$ generate on the space $C_{\Delta}$ invariant under $T(t)$ subspaces $P_{1}, P_{2}, Q$. Subspaces $P_{1}, P_{2}$ correspond to the initial values of all those solutions of 
(2.1) which are in the form $q(t) e^{\lambda t}$, where $\lambda \in \Lambda_{i}, i=1,2, q(t)$ is a polynomial in $t$. They are finite dmensional. Let their dimensions be equal to $n_{1}, n_{2}$ respectively.

We denote by $\Phi_{i}(\theta), \Delta \leq \theta \leq 0$ is a basis of $P_{i}$, and $\Psi_{i}, 0 \leq \theta \leq \Delta$ is a basis of $P_{i}^{*} \subset C[0, \Delta]$ of the initial values of solutions of the adjoint to (2.1) system

$$
\frac{d y}{d t}=A^{*} y_{t}, \quad A^{*} \psi=-\int_{-\Delta}^{0}\left[d \eta_{1}^{T}(\theta)\right] \psi(-\theta) .
$$

For elements $\varphi \in C[-\Delta, 0], \psi \in C[0, \Delta]$ we define the scalar product by

$$
(\psi, \varphi)=\psi^{T}(0) \varphi(0)-\int_{-\Delta}^{0} \int_{0}^{\theta} \psi(\xi-\theta)\left[d \eta_{1}(\theta)\right] \varphi(\xi) d \xi .
$$

It is known [11] that $n_{i} \times n_{i}$ matrix $\left(\Psi_{i}, \Phi_{i}\right)$ is nonsingular and we can take that $\left(\Psi_{i}, \Phi_{i}\right)=E$. Let $B_{i}$ denote $n_{i} \times n_{i}$ matrix such that

$$
\frac{d}{d \theta} \Phi_{i}(\theta)=\Phi_{i}(\theta) B, \quad i=1,2, \quad \theta \in[-\Delta, 0] .
$$

The space $C_{\Delta}$ can be decomposed into direct sum $C_{\Delta}=P_{1}+P_{2}+Q$. Every element $x_{t} \in C_{\Delta}$ can be represented in the form $[11,12]$

$$
\begin{gathered}
x_{t}=\Phi_{1} u_{1}(t)+\Phi_{2} u_{2}(t)+z_{t}, \\
u_{1}(t)=\left(\Psi_{1}, x_{t}\right), \quad u_{2}(t)=\left(\Psi_{2}, x_{t}\right), \quad z_{t} \in Q, \quad\left(\Psi_{i}, z_{t}\right)=0, i=1,2 .
\end{gathered}
$$

Define matrices

$$
X_{0}(\theta)=\left\{\begin{array}{ll}
0, & -\Delta \leq \theta<0, \\
E, & \theta=0,
\end{array} \quad Y_{0}(\theta)= \begin{cases}0, & -\varepsilon \Delta \leq \theta<0, \\
E, & \theta=0\end{cases}\right.
$$

and shift operator $V(t, \sigma)$ for equation

$$
\varepsilon \frac{d y}{d t}=\int_{-\Delta}^{0}\left[d \eta_{2}(t, \theta)\right] y(t+\varepsilon \theta) .
$$

Changing variables (2.2) in system (1.1) and using the variation of constants formula [12] we get the equivalent system of differential and integral equations

$$
\begin{gathered}
\frac{d u_{i}}{d t}=B_{i} u_{i}+F_{i}\left(t, u_{1}, u_{2}, y_{t}, z_{t}, \varepsilon\right), \quad i=1,2, \\
z_{t}=T(t-\sigma) z_{\sigma}+\int_{\sigma}^{t} T(t-s) X_{0}^{Q} F\left(s, u_{1}, u_{2}, y_{t}, z_{t}, \varepsilon\right) d s \\
y_{t}=V(t, \sigma) y_{\sigma}+\frac{1}{\varepsilon} \int_{\sigma}^{t} V(t, s) Y_{0} G\left(s, u_{1}, u_{2}, y_{t}, z_{t}, \varepsilon\right) d s
\end{gathered}
$$

where

$$
F\left(t, u_{1}, u_{2}, y_{t}, z_{t}, \varepsilon\right)=f\left(t, \Phi_{1} u_{1}+\Phi_{2} u_{2}+z_{t}, y_{t}, \varepsilon\right),
$$




$$
\begin{gathered}
F_{i}\left(t, u_{1}, u_{2}, y_{t}, z_{t}, \varepsilon\right)=\Psi_{i}^{T}(0) F\left(t, u_{1}, u_{2}, y_{t}, z_{t}, \varepsilon\right), i=1,2, \\
G\left(t, u_{1}, u_{2}, y_{t}, z_{t}, \varepsilon\right)=g\left(t, \Phi_{1} u_{1}+\Phi_{2} u_{2}+z_{t}, y_{t}, \varepsilon\right), \\
X_{0}^{P_{i}}=\Phi_{i} \Psi_{i}^{T}(0), i=1,2, \quad X_{0}^{Q}=X_{0}-X_{0}^{P_{1}}-X_{0}^{P_{2}} .
\end{gathered}
$$

The integrals in (2.4) for each $\theta$ are understood as the integrals in Euclidean spaces $R^{n}$ and $R^{m}$.

From the definition of the sets $\Lambda_{i}, i=\overline{1,3}$ and under assumption II there exist positive constants $K_{1}, K_{2}, \alpha$ such that following inequalities are valid $[12,13]$ :

$$
\begin{gathered}
\left|e^{B_{1} t}\right| \leq K_{1} e^{\alpha t}, \quad t \leq 0, \\
\left|e^{B_{2} t}\right| \leq K_{1} e^{\frac{\alpha}{4}|t|}, \quad t \in R, \\
\left|T(t) \varphi^{Q}\right| \leq K_{1} e^{-\alpha t}\left|\varphi^{Q}\right|, \quad t \geq 0, \\
|V(t, \sigma) \xi| \leq K_{2} e^{-\frac{\mu}{\varepsilon}(t-\sigma)}|\xi|, \quad t \geq \sigma, \xi \in C_{\varepsilon \Delta} .
\end{gathered}
$$

\section{Existence of the center and center-unstable integral manifolds}

Definition 1. A set of points $M \subset R \times R^{n_{1}} \times R^{n_{2}} \times Q \times C_{\varepsilon \Delta}$ is said to be an integral manifold of system (2.4) if for each $\varepsilon \in\left[0, \varepsilon_{0}\right]$ and any point $\left(t_{0}, u_{10}, u_{20}, z_{t_{0}}, y_{t_{0}}\right) \in M$ it follows that $\left(t, u_{1}(t), u_{2}(t), z_{t}, y_{t}\right) \in M$ for all $t \geq t_{0}$, where $\left(u_{1}(t), u_{2}(t), z_{t}, y_{t}\right)$ is the solution of system (2.4) with the initial values $\left(t_{0}, u_{10}, u_{20}, z_{t_{0}}, y_{t_{0}}\right)$.

Theorem 1. Let conditions I-II hold. Then there exist positive constants $\rho_{0}, \eta_{0}$ such that for all $0<\rho<\rho_{0}, 0<\eta<\eta_{0}$ and sufficiently small $\varepsilon$ the system (2.4) has the center and center-unstable integral manifolds

$$
\begin{gathered}
M^{*}=\left\{\left(t, u_{1}, u_{2}, z, y\right): t \in R, u_{1}=r^{*}\left(t, u_{2}, \varepsilon\right), u_{2} \in R^{n_{2}},\right. \\
\left.z=v^{*}\left(t, u_{2}, \varepsilon\right), y=w^{*}\left(t, u_{2}, \varepsilon\right)\right\}, \\
M^{*-}=\left\{\left(t, u_{1}, u_{2}, z, y\right): t \in R, u_{1} \in R^{n_{1}}, u_{2} \in R^{n_{2}},\right. \\
\left.z=v^{*-}\left(t, u_{1}, u_{2}, \varepsilon\right), y=w^{*-}\left(t, u_{1}, u_{2}, \varepsilon\right)\right\},
\end{gathered}
$$

where functions $r^{*}, v^{*}, w^{*}, v^{*-}, w^{*-}$ are continuous with respect to all variables and satisfy the Lipschitz condition by $u_{1}, u_{2}$ with constant $\eta$ and not exceeding $\rho$.

Proof. The existence of the center manifold of system (2.4) was studied in [14]. Now we prove that system (2.4) has center-unstable integral manifold $M^{*-}$.

Define the sets of functions

$$
\begin{gathered}
S_{z}=\left\{v: R \times R^{n_{1}} \times R^{n_{2}} \times\left[0, \varepsilon_{0}\right] \rightarrow Q\right\}, \\
S_{y}=\left\{w: R \times R^{n_{1}} \times R^{n_{2}} \times\left[0, \varepsilon_{0}\right] \rightarrow C_{\varepsilon \Delta}\right\},
\end{gathered}
$$

which are bounded by $\rho$ and satisfy the Lipschitz condition by $u_{1}, u_{2}$ with constant $\eta$. 
Consider the following system for any $v \in S_{z}, w \in S_{y}$

$$
\frac{d u_{i}}{d t}=B_{i} u_{i}+F_{i}\left(t, u_{1}, u_{2}, v\left(t, u_{1}, u_{2}, \varepsilon\right), w\left(t, u_{1}, u_{2}, \varepsilon\right), \varepsilon\right), i=1,2 .
$$

Due to the conditions of functions $f, v, w$ the following inequalities hold

$$
\begin{aligned}
& \mid F_{i}\left(t, u_{1}, u_{2}, v\left(t, u_{1}, u_{2}, \varepsilon\right), w\left(t, u_{1}, u_{2}, \varepsilon\right), \varepsilon\right)- \\
- & F_{i}\left(t, \bar{u}_{1}, \bar{u}_{2}, v\left(t, \bar{u}_{1}, \bar{u}_{2}, \varepsilon\right), w\left(t, \bar{u}_{1}, \bar{u}_{2}, \varepsilon\right), \varepsilon\right) \mid \leq \\
\leq & L(\varepsilon) m_{i}\left(\nu_{1}+\nu_{2}+2 \eta\right)\left(\left|u_{1}-\bar{u}_{1}\right|+\left|u_{2}-\bar{u}_{2}\right|\right),
\end{aligned}
$$

where $m_{i}=\left|\Psi_{i}^{T}(0)\right|, \nu_{i}=\left|\Phi_{i}\right|, i=1,2$.

Thus, for every point $\left(u_{10}, u_{20}\right) \in R^{n_{1}} \times R^{n_{2}}$ system (3.1) has a unique solution $u_{1}(t)=U_{1}\left(t, t_{0}, u_{10}, \varepsilon, v, w\right), u_{2}(t)=U_{2}\left(t, t_{0}, u_{20}, \varepsilon, v, w\right)$ such that $u_{1}\left(t_{0}\right)=u_{10}$, $u_{2}\left(t_{0}\right)=u_{20}$.

Lemma 1. Let conditions I-II hold. Then for all sufficient small $\varepsilon$ the following inequality is valid

$\left|U_{1}-\bar{U}_{1}\right|+\left|U_{2}-\bar{U}_{2}\right| \leq K_{1} e^{-\frac{\alpha}{2}(t-s)}\left(\left|u_{1}-\bar{u}_{1}\right|+\left|u_{2}-\bar{u}_{2}\right|+|| v-\bar{v}||+\| w-\bar{w}||\right), t \leq s$.

The proof of Lemma 1 can be easily obtained using the Gronwal inequality and properties of functions $f, v, w$.

Consider now the set $S=S_{z} \times S_{y}$ of functions $(v, w)$ with the norm $\|(v, w)\|=$ $\max (\|v\|,\|w\|)$. Define in set $S$ the operator

$$
H(v, w)=\left(H_{t, u_{1}, u_{2}}^{1}(v, w), H_{t, u_{1}, u_{2}}^{2}(v, w)\right),
$$

where

$$
\begin{gathered}
H_{t, u_{1}, u_{2}}^{1}(v, w)=\int_{-\infty}^{t} T(t-s) X_{0}^{Q} F\left(s, u_{1}(s), u_{2}(s),\right. \\
\left.v\left(s, u_{1}, u_{2}, \varepsilon\right), w\left(s, u_{1}, u_{2}, \varepsilon\right), \varepsilon\right) d s \\
H_{t, u_{1}, u_{2}}^{2}(v, w)=\frac{1}{\varepsilon} \int_{-\infty}^{t} V(t, s) Y_{0} G\left(s, u_{1}(s), u_{2}(s),\right. \\
\left.v\left(s, u_{1}, u_{2}, \varepsilon\right), w\left(s, u_{1}, u_{2}, \varepsilon\right), \varepsilon\right) d s .
\end{gathered}
$$

It follows from inequalities (2.5) and condition II that the integrals on the right side of $(3.2),(3.3)$ converge uniformly with respect to $\theta \in[-\Delta, 0]$.

Consider any sequence of numbers $t_{n}<t$ such that $t_{n} \rightarrow-\infty$ when $n \rightarrow \infty$. It follows from (2.4) that

$$
\begin{aligned}
& A_{n}=\int_{t_{n}}^{t} T(t-s) Y_{0}^{Q} F\left(s, u_{1}, u_{2}, v, w, \varepsilon\right) d s \in Q, \\
& B_{n}=\frac{1}{\varepsilon} \int_{t_{n}}^{t} V(t, s) Y_{0} G\left(s, u_{1}, u_{2}, v, w, \varepsilon\right) d s \in C_{\varepsilon \Delta} .
\end{aligned}
$$


Then $H_{t, u_{1}, u_{2}}^{1}(v, w) \in Q, H_{t, u_{1}, u_{2}}^{2}(v, w) \in C_{\varepsilon \Delta}$ since $A_{n} \rightarrow H_{t, u_{1}, u_{2}}^{1}(v, w), B_{n} \rightarrow$ $H_{t, u_{1}, u_{2}}^{2}(v, w)$ when $n \rightarrow \infty$ uniformly with respect to $\theta \in[-\Delta, 0]$.

By means of similar arguments to those in [14] one can easily make sure that the following statement is valid.

Lemma 2. Let conditions I-II hold. Then for all sufficiently small $\varepsilon$ the operator $H$ is a contraction mapping of $S$ into itself.

Denote by $\left(v^{*-}, w^{*-}\right)$ the unique fixed point of the operator $H$. Let $u_{1}(t)=$ $U_{1}\left(t, t_{0}, u_{10}, \varepsilon, v^{*-}, w^{*-}\right), u_{2}(t)=U_{2}\left(t, t_{0}, u_{20}, \varepsilon, v^{*-}, w^{*-}\right)$ be a solution of system (3.1). Then functions $v^{*-}, w^{*-}$ satisfy such system

$$
\begin{gathered}
v^{*-}\left(t, u_{1}(t), u_{2}(t), \varepsilon\right)=\int_{-\infty}^{t} T(t-s) X_{0}^{Q} F\left(s, u_{1}(s), u_{2}(s),\right. \\
\left.v^{*-}\left(s, u_{1}(s), u_{2}(s), \varepsilon\right), w^{*-}\left(s, u_{1}(s), u_{2}(s), \varepsilon\right), \varepsilon\right) d s, \\
w^{*-}\left(t, u_{1}(t), u_{2}(t), \varepsilon\right)=\frac{1}{\varepsilon} \int_{-\infty}^{t} V(t, s) Y_{0} G\left(s, u_{1}(s), u_{2}(s),\right. \\
\left.v^{*-}\left(s, u_{1}(s), u_{2}(s), \varepsilon\right), w^{*-}\left(s, u_{1}(s), u_{2}(s), \varepsilon\right), \varepsilon\right) d s .
\end{gathered}
$$

Let $\left(t_{0}, u_{10}, u_{20}\right) \in R \times R^{n_{1}} \times R^{n_{2}}$ be an arbitrary point. Using the following identity for solutions of system $(3.1)$

$$
U_{i}\left(s, t_{0}, u_{i 0}, \varepsilon, v, w\right)=U_{i}\left(s, t, U_{i}\left(t, t_{0}, u_{i 0}, \varepsilon, v, w\right), \varepsilon, v, w\right), i=1,2
$$

and also the property of the operators $T, V$

$$
T(t-s)=T\left(t-t_{0}\right) T\left(t_{0}-s\right), \quad V(t, s)=V\left(t, t_{0}\right) V\left(t_{0}, s\right)
$$

we rewrite system (3.4) in the form

$$
\begin{aligned}
v^{*-}\left(t, u_{1}, u_{2}, \varepsilon\right)= & T\left(t-t_{0}\right) v^{*-}\left(t_{0}, u_{10}, u_{20}, \varepsilon\right)+\int_{t_{0}}^{t} T(t-s) X_{0}^{Q} F\left(s, u_{1}, u_{2},\right. \\
& \left.v^{*-}\left(s, u_{1}, u_{2}, \varepsilon\right), w^{*-}\left(s, u_{1}, u_{2}, \varepsilon\right), \varepsilon\right) d s, \\
w^{*-}\left(t, u_{1}, u_{2}, \varepsilon\right)= & V\left(t, t_{0}\right) w^{*-}\left(t_{0}, u_{10}, u_{20}, \varepsilon\right)+\frac{1}{\varepsilon} \int_{t_{0}}^{t} V(t, s) Y_{0} G\left(s, u_{1}, u_{2},\right. \\
& \left.v^{*-}\left(s, u_{1}, u_{2}, \varepsilon\right), w^{*-}\left(s, u_{1}, u_{2}, \varepsilon\right), \varepsilon\right) d s .
\end{aligned}
$$

It follows from (3.5) that functions $v^{*-}\left(s, u_{1}, u_{2}, \varepsilon\right), w^{*-}\left(s, u_{1}, u_{2}, \varepsilon\right)$ satisfy the third and fourth equations of system (2.4) for all $t \geq t_{0}$. Thus, the set $M^{*-}$ is an integral manifold for (2.4). 
Corollary 1. The flow of the initial system (1.1) on the center-stable manifold $M^{*-}$ is governed by the system of ordinary differential equations

$$
\frac{d u_{i}}{d t}=B_{i} u_{i}+F_{i}\left(t, u_{1}, u_{2}, v^{*-}\left(t, u_{1}, u_{2}, \varepsilon\right), w^{*-}\left(t, u_{1}, u_{2}, \varepsilon\right), \varepsilon\right), i=1,2,
$$

which is regular and has no time lag.

\section{Existence of the stable and center-stable integral manifolds}

Further we suppose that such equalities are valid:

$$
f(t, 0,0, \varepsilon)=0, \quad g(t, 0,0, \varepsilon)=0 .
$$

Theorem 1. Let conditions $I-I I$ be valid. Then there exist positive constants $l_{0}, N$ such that for all $0<l<l_{0}$ and sufficiently small $\varepsilon$ system (2.4) has the stable and center-stable manifolds

$$
\begin{gathered}
M^{+}=\left\{\left(t, u_{1}, u_{2}, z, y\right): u_{1}=r_{1}^{+}(t, z, y, \varepsilon),\right. \\
\left.u_{2}=r_{2}^{+}(t, z, y, \varepsilon), z \in Q, y \in C_{\varepsilon \Delta}\right\}, \\
M^{*+}=\left\{\left(t, u_{1}, u_{2}, z, y\right): u_{1}=r^{*+}\left(t, u_{1}, z, y, \varepsilon\right), u_{2} \in R^{n_{2}}, z \in Q, y \in C_{\varepsilon \Delta}\right\},
\end{gathered}
$$

where functions $r_{1}^{+}, r_{2}^{+}, r^{*+}$ are continuous with respect to all variables and satisfy the Lipschitz condition by $u_{2}, y, z$ with constant $l$.

The following estimates are valid for the arbitrary solution of the system (2.4), whose initial values lie on a stable manifold $M^{+}$, when $t \geq t_{0}$ :

$$
\begin{gathered}
\left|u_{i}\right| \leq N\left(\left|y_{t_{0}}\right|+\left|z_{t_{0}}\right|\right) e^{-\frac{\alpha}{2}\left(t-t_{0}\right)}, i=1,2, \\
\left|z_{t}\right| \leq N\left(\left|y_{t_{0}}\right|+\left|z_{t_{0}}\right|\right) e^{-\frac{\alpha}{2}\left(t-t_{0}\right)}, \\
\left|y_{t}\right| \leq N\left(\left|y_{t_{0}}\right|+\left|z_{t_{0}}\right|\right) e^{-\frac{\alpha}{2}\left(t-t_{0}\right)} .
\end{gathered}
$$

Proof. Denote by

$$
\Omega:=\left\{r_{i}: R \times Q \times C_{\varepsilon \Delta} \times\left[0, \varepsilon_{1}\right] \rightarrow R^{n_{i}}\right\}, i=1,2
$$

the sets of all continuous functions with respect to all variables, which satisfy the Lipschitz condition on $z, y$ with constant $l$ and condition $r_{i}(t, 0,0, \varepsilon)=0$. For arbitrary $r_{1} \in \Omega_{1}, r_{2} \in \Omega_{2}$ we consider the following system

$$
\begin{gathered}
z_{t}=T\left(t-t_{0}\right) z_{t_{0}}+\int_{t_{0}}^{t} T(t-s) X_{0}^{Q} F\left[s, z_{s}, y_{s}, \varepsilon\right] d s, \\
y_{t}=V\left(t, t_{0}\right) y_{t_{0}}+\frac{1}{\varepsilon} \int_{t_{0}}^{t} V(t, s) Y_{0} G\left[s, z_{s}, y_{s}, \varepsilon\right] d s
\end{gathered}
$$

where

$$
\begin{aligned}
& F\left[s, z_{s}, y_{s}, \varepsilon\right]=F\left(s, r_{1}\left(s, z_{s}, y_{s}, \varepsilon\right), r_{2}\left(s, z_{s}, y_{s}, \varepsilon\right), z_{s}, y_{s}, \varepsilon\right) \\
& G\left[s, z_{s}, y_{s}, \varepsilon\right]=G\left(s, r_{1}\left(s, z_{s}, y_{s}, \varepsilon\right), r_{2}\left(s, z_{s}, y_{s}, \varepsilon\right), z_{s}, y_{s}, \varepsilon\right)
\end{aligned}
$$


Let us prove the existence of the solution of system (4.2) with the help of the successive approximations method

$$
\begin{gathered}
z_{t}^{0}=0, \quad y_{t}^{0}=0 \\
z_{t}^{j+1}=T\left(t-t_{0}\right) z_{t_{0}}+\int_{t_{0}}^{t} T(t-s) X_{0}^{Q} F\left[s, z_{s}^{j}, y_{s}^{j}, \varepsilon\right] d s \\
y_{t}^{j+1}=V\left(t, t_{0}\right) y_{t_{0}}+\frac{1}{\varepsilon} \int_{t_{0}}^{t} V(t, s) Y_{0} G\left[s, z_{s}^{j}, y_{s}^{j}, \varepsilon\right] d s, \quad j=0,1, \ldots
\end{gathered}
$$

With the help of the mathematical induction method we ensure that the following inequalities are valid:

$$
\begin{aligned}
& \left|z_{t}^{m+1}-z_{t}^{m}\right| \leq \frac{\bar{K}}{2^{m}} e^{-\frac{\alpha}{2}\left(t-t_{0}\right)}\left(\left|z_{t_{0}}\right|+\left|y_{t_{0}}\right|\right), \\
& \left|y_{t}^{m+1}-y_{t}^{m}\right| \leq \frac{\bar{K}}{2^{m}} e^{-\frac{\alpha}{2}\left(t-t_{0}\right)}\left(\left|z_{t_{0}}\right|+\left|y_{t_{0}}\right|\right),
\end{aligned}
$$

where $\bar{K}=\max \left(K_{1}, K_{2}\right), m=0,1, \ldots$

From estimates (2.5) for $\varepsilon<\frac{2 \mu}{\alpha}$ we obtain that the inequalities (4.3) are valid for $m=0$. Suppose that (4.3) are valid for $m=j$. Then obtain

$$
\begin{aligned}
\left|z_{t}^{j+1}-z_{t}^{j}\right| \leq & \int_{t_{0}}^{t} K_{1} e^{-\alpha(t-s)} L(\varepsilon)\left(\nu_{1} l+\nu_{2} l+1\right)\left(\left|z_{t}^{j}-z_{t}^{j-1}\right|+\left|y_{t}^{j}-y_{t}^{j-1}\right|\right) d s \leq \\
& \leq \frac{\bar{K}}{2^{j-1}} \frac{4 L(\varepsilon)\left(\nu_{1} l+\nu_{2} l+1\right)}{\alpha}\left(\left|z_{t_{0}}\right|+\left|y_{t_{0}}\right|\right) e^{-\frac{\alpha}{2}\left(t-t_{0}\right)}, \\
\left|y_{t}^{j+1}-y_{t}^{j}\right| \leq & \int_{t_{0}}^{t} K_{2} e^{-\frac{\mu}{\varepsilon}(t-s)} L(\varepsilon)\left(\nu_{1} l+\nu_{2} l+1\right)\left(\left|z_{t}^{j}-z_{t}^{j-1}\right|+\left|y_{t}^{j}-y_{t}^{j-1}\right|\right) d s \leq \\
& \leq \frac{\bar{K}}{2^{j-1}} \frac{4 L(\varepsilon)\left(\nu_{1} l+\nu_{2} l+1\right)}{2 \mu-\varepsilon \alpha}\left(\left|z_{t_{0}}\right|+\left|y_{t_{0}}\right|\right) e^{-\frac{\alpha}{2}\left(t-t_{0}\right)} .
\end{aligned}
$$

For $\varepsilon<\frac{\mu}{\alpha}$ and $L(\varepsilon)<\frac{\alpha}{8 \bar{K}\left(\nu_{1} l+\nu_{2} l+1\right)}$ we obtain that (4.3) are valid for $m=j+1$. Thus, inequalities (4.3) are valid for all natural $m$.

It follows from (4.3) that sequences $z_{t}^{m}, y_{t}^{m}$ uniformly converge to the solutions $z_{t}\left(t_{0}, z_{t_{0}}, y_{t_{0}}, r_{1}, r_{2}\right), y_{t}\left(t_{0}, z_{t_{0}}, y_{t_{0}}, r_{1}, r_{2}\right)$ of the system (4.2). After summation of inequaities (4.3) on $m$, it is obtained the following uniform estimates:

$$
\begin{aligned}
& \left|z_{t}\left(t_{0}, z_{t_{0}}, y_{t_{0}}, r_{1}, r_{2}\right)\right| \leq 2 \bar{K}\left(\left|z_{t_{0}}\right|+\left|y_{t_{0}}\right|\right) e^{-\frac{\alpha}{2}\left(t-t_{0}\right)}, \\
& \left|y_{t}\left(t_{0}, z_{t_{0}}, y_{t_{0}}, r_{1}, r_{2}\right)\right| \leq 2 \bar{K}\left(\left|z_{t_{0}}\right|+\left|y_{t_{0}}\right|\right) e^{-\frac{\alpha}{2}\left(t-t_{0}\right)},
\end{aligned}
$$

for $t \geq t_{0}$. 
Denote by $z_{t}\left(t_{0}, \bar{z}_{t_{0}}, \bar{y}_{t_{0}}, r_{1}, r_{2}\right), y_{t}\left(t_{0}, \bar{z}_{t_{0}}, \bar{y}_{t_{0}}, r_{1}, r_{2}\right)$ the solution of system (4.2) with initial value $\left(\bar{z}_{t_{0}}, \bar{y}_{t_{0}}\right)$. Similarly to (4.3), (4.4) one can show that the following estimations are valid:

$$
\begin{gathered}
\left|z_{t}\left(t_{0}, z_{t_{0}}, y_{t_{0}}, r_{1}, r_{2}\right)-z_{t}\left(t_{0}, \bar{z}_{t_{0}}, \bar{y}_{t_{0}}, r_{1}, r_{2}\right)\right| \leq \\
2 \bar{K}\left(\left|z_{t_{0}}-\bar{z}_{t_{0}}\right|+\left|y_{t_{0}}-\bar{y}_{t_{0}}\right|\right) e^{-\frac{\alpha}{2}\left(t-t_{0}\right)}, \\
\left|y_{t}\left(t_{0}, z_{t_{0}}, y_{t_{0}}, r_{1}, r_{2}\right)-y_{t}\left(t_{0}, \bar{z}_{t_{0}}, \bar{y}_{t_{0}}, r_{1}, r_{2}\right)\right| \leq \\
2 \bar{K}\left(\left|z_{t_{0}}-\bar{z}_{t_{0}}\right|+\left|y_{t_{0}}-\bar{y}_{t_{0}}\right|\right) e^{-\frac{\alpha}{2}\left(t-t_{0}\right)},
\end{gathered}
$$

for $t \geq t_{0}$.

Let us denote by $\Omega=\Omega_{1} \times \Omega_{2}$ the set of function $\left(r_{1}, r_{2}\right)$ with the norm

$$
\left\|\left(r_{1}, r_{2}\right)\right\|=\max \left(\left\|r_{1}\right\|,\left\|r_{2}\right\|\right), \quad\|\cdot\|=\sup _{t, z, y, \varepsilon}|\cdot| .
$$

Let define the operator

$$
H\left(r_{1}, r_{2}\right)=\left(H_{r_{1}, r_{2}}^{1}\left(t_{0}, z_{t_{0}}, y_{t_{0}}, \varepsilon\right), H_{r_{1}, r_{2}}^{2}\left(t_{0}, z_{t_{0}}, y_{t_{0}}, \varepsilon\right)\right),
$$

where

$$
H_{r_{1}, r_{2}}^{i}\left(t_{0}, z_{t_{0}}, y_{t_{0}}, \varepsilon\right)=-\int_{t_{0}}^{\infty} e^{B_{i}(t-s)} F\left[s, z_{s}, y_{s}, \varepsilon\right] d s, \quad i=1,2 .
$$

From estimates (2.5) and inequalities (4.4), (4.5) it follows that for sufficiently small $\varepsilon$ the operator $H$ maps $\Omega$ into itself.

Let $\left(r_{1}, r_{2}\right),\left(\bar{r}_{1}, \bar{r}_{2}\right) \in \Omega$. Using the estimates (2.5), (4.5) and properties of functions $f, r_{1}, r_{2}$, we obtain

$$
\begin{gathered}
\left|H_{r_{1}, r_{2}}^{i}\left(t_{0}, z_{t_{0}}, y_{t_{0}}, \varepsilon\right)-H_{\bar{r}_{1}, \bar{r}_{2}}^{i}\left(t_{0}, \bar{z}_{t_{0}}, \bar{y}_{t_{0}}, \varepsilon\right)\right| \leq \\
\leq \frac{\bar{K}\left(\nu_{1}+\nu_{2}\right)}{\alpha} L(\varepsilon)\left(\left\|r_{1}-\bar{r}_{1}\right\|+\left\|r_{2}-\bar{r}_{2}\right\|\right) .
\end{gathered}
$$

Thus, operator $H$ is a contraction on $\Omega$ if

$$
L<\frac{\alpha}{\bar{K}\left(\nu_{1}+\nu_{2}\right)} .
$$

Let us denote by $\left(r_{1}^{+}, r_{2}^{+}\right)$the unique fixed point of $H$ on $\Omega$. We are going to prove that $M^{+}$is an integral manifold. Let $\left(z_{t}, y_{t}\right)$ be the solution of the system (4.2), where $r_{i}=r_{i}^{+}, i=1,2$. Then functions $r_{i}^{+}, i=1,2$ satisfy equations

$$
r_{i}^{+}=-\int_{t}^{\infty} e^{B_{i}(t-s)} F\left[s, z_{s}, y_{s}, \varepsilon\right] d s, \quad i=1,2 .
$$

Differentiating these equations we obtain that functions $r_{i}^{+}, i=1,2$ satisfy the first and the second equations of system (2.4). Thus, the set $M^{+}$is an integral manifold for system (2.4).

From (4.5) and properties of the functions $r_{1}^{+}, r_{2}^{+}$it follows that estimates (4.1) are valid for $N=\max (2 \bar{K}, 4 l \bar{K})$. The existence of the center-stable manifold $M^{*+}$ is proved similarly to the case of stable manifold. 


\section{The stability problem}

In this item we study the behaviour of solutions of system (1.1) when $t \rightarrow \infty$.

Theorem 1. Let the conditions I-II are valid. Then for all sufficiently small $\varepsilon$ and arbitrary solution $\left(u_{1}(t), u_{2}(t), z_{t}, y_{t}\right)$ of system (2.4) there exists a solution

$$
\left(h_{1}(t), h_{2}(t), v^{*-}\left(t, h_{1}, h_{2}, \varepsilon\right), w^{*-}\left(t, h_{1}, h_{2}, \varepsilon\right)\right)
$$

of system (2.4), starting from a center-unstable manifold that following inequalities hold

$$
\begin{gathered}
\left|u_{i}(t)-h_{i}(t)\right| \leq K_{3} \varphi e^{-\frac{\alpha}{2}\left(t-t_{0}\right)}, \quad i=1,2 \\
\left|z_{t}-v^{*-}\left(t, h_{1}, h_{2}, \varepsilon\right)\right| \leq K_{3} \varphi e^{-\frac{\alpha}{2}\left(t-t_{0}\right)} \\
\left|y_{t}-w^{*-}\left(t, h_{1}, h_{2}, \varepsilon\right)\right| \leq K_{3} \varphi e^{-\frac{\alpha}{2}\left(t-t_{0}\right)}
\end{gathered}
$$

where $\varphi=\left|z_{t_{0}}-v^{*-}\left(t, h_{1}\left(t_{0}\right), h_{2}\left(t_{0}\right), \varepsilon\right)\right|+\left|y_{t_{0}}-w^{*-}\left(t, h_{1}\left(t_{0}\right), h_{2}\left(t_{0}\right), \varepsilon\right)\right|, K_{3}>0$.

Proof. Let consider arbitrary solution $\left(u_{1}(t), u_{2}(t), z_{t}, y_{t}\right)$ of system (2.4) with initial values $\left(t_{0}, u_{10}, u_{20}, z_{t_{0}}, y_{t_{0}}\right)$ and a solution $\left(h_{1}(t), h_{2}(t), \xi_{t}, \psi_{t}\right)$ of system (2.4) with initial values $\left(c_{1}, c_{2}, v^{*-}\left(t_{0}, c_{1}, c_{2}, \varepsilon\right), w^{*-}\left(t_{0}, c_{1}, c_{2}, \varepsilon\right)\right)$, where $c_{1}=h_{1}\left(t_{0}\right), c_{2}=h_{2}\left(t_{0}\right)$. Changing the variables in system (2.4)

$$
p_{1}(t)=u_{1}(t)-h_{1}(t), \quad p_{2}(t)=u_{2}(t)-h_{2}(t), \quad q_{t}=z_{t}-\xi_{t}, \quad \omega_{t}=y_{t}-\psi_{t},
$$

we obtain the following system

$$
\begin{gathered}
\frac{d p_{i}}{d t}=B_{i} p_{i}+\bar{F}_{i}\left(t, p_{1}, p_{2}, q_{t}, \omega_{t}, \varepsilon\right), \quad i=1,2, \\
q_{t}=T\left(t-t_{0}\right) q_{t_{0}}+\int_{t_{0}}^{t} T(t-s) X_{0}^{Q} \bar{F}\left(s, p_{1}, p_{2}, q_{s}, \omega_{s}, \varepsilon\right) d s \\
\omega_{t}=V\left(t, t_{0}\right) \omega_{t_{0}}+\frac{1}{\varepsilon} \int_{t_{0}}^{t} V(t, s) Y_{0} \bar{G}\left(s, p_{1}, p_{2}, q_{s}, \omega_{s}, \varepsilon\right) d s
\end{gathered}
$$

where

$$
\bar{F}_{i}=F_{i}\left(t, p_{1}+h_{1}, p_{2}+h_{2}, q_{t}+\xi_{t}, \omega_{t}+\psi_{t}, \varepsilon\right)-F_{i}\left(t, h_{1}, h_{2}, \xi_{t}, \psi_{t}, \varepsilon\right), i=1,2
$$

and functions $\bar{F}, \bar{G}$ have the same form.

According to theorem 1, the system (5.3) has a stable integral manifold. This manifold is described by functions $p_{i}=r_{i}^{+}\left(t, q_{t}, \omega_{t}, \varepsilon\right), i=1,2$, which satisfy the following conditions

$$
\begin{gathered}
r_{i}^{+}(t, 0,0, \varepsilon)=0 \\
\left|r_{i}^{+}\left(t, q_{t}, \omega_{t}, \varepsilon\right)-r_{i}^{+}\left(t, \bar{q}_{t}, \bar{\omega}_{t}, \varepsilon\right)\right| \leq l\left(\left|q_{t}-\bar{q}_{t}\right|+\left|\omega_{t}-\bar{\omega}_{t}\right|\right) .
\end{gathered}
$$

For arbitrary solution $\left(p_{1}, p_{2}, q_{t}, \omega_{t}\right)$ of system (5.3), which belongs to $M^{+}$, it follows that

$$
\begin{gathered}
\left|p_{i}(t)\right| \leq N e^{-\frac{\alpha}{2}\left(t-t_{0}\right)}\left(\left|q_{t_{0}}\right|+\left|\omega_{t_{0}}\right|\right), \quad i=1,2, \\
\left|q_{t}\right| \leq N e^{-\frac{\alpha}{2}\left(t-t_{0}\right)}\left(\left|q_{t_{0}}\right|+\left|\omega_{t_{0}}\right|\right),
\end{gathered}
$$




$$
\left|\omega_{t}\right| \leq N e^{-\frac{\alpha}{2}\left(t-t_{0}\right)}\left(\left|q_{t_{0}}\right|+\left|\omega_{t_{0}}\right|\right)
$$

Let us now show that representations (5.2) are valid if solutions $\left(p_{1}(t), p_{2}(t), q_{t}, \omega_{t}\right)$ are on the stable manifold $M^{+}$. It is sufficient to show that (5.2) holds for $t=t_{0}$. In this case we obtain

$$
\begin{aligned}
& r_{1}^{+}\left(t_{0}, q_{t_{0}}, \omega_{t_{0}}, \varepsilon\right)=u_{10}-c_{1}, \quad r_{2}^{+}\left(t_{0}, q_{t_{0}}, \omega_{t_{0}}, \varepsilon\right)=u_{20}-c_{2}, \\
& q_{t_{0}}=z_{t_{0}}-v^{*-}\left(t_{0}, c_{1}, c_{2}, \varepsilon\right), \quad \omega_{t_{0}}=y_{t_{0}}-w^{*-}\left(t_{0}, c_{1}, c_{2}, \varepsilon\right) .
\end{aligned}
$$

Next we show that system (5.5) has the solution with respect to $\left(c_{1}, c_{2}, q_{t_{0}}, w_{t_{0}}\right)$ for any $\left(u_{10}, u_{20}, z_{t_{0}}, \omega_{t_{0}}\right)$.

Let $S$ denote the sphere in the space $R^{n_{1}} \times R^{n_{2}}$ which is defined by

$$
\left|c_{1}-u_{10}\right|+\left|c_{2}-u_{20}\right| \leq 4 l\left(\left|z_{t_{0}}-v^{*-}\left(t_{0}, u_{10}, u_{20}, \varepsilon\right)\right|+\left|y_{t_{0}}-w^{*-}\left(t_{0}, u_{10}, u_{20}, \varepsilon\right)\right|\right) \text {. }
$$

Let consider the operator $J=\left(J_{1}, J_{2}\right)$ on $S$, where

$$
J_{i}\left(c_{1}, c_{2}\right)=u_{i 0}-r_{i}^{*}\left(t_{0}, z_{t_{0}}-v^{*-}\left(t_{0}, c_{1}, c_{2}, \varepsilon\right), y_{t_{0}}-w^{*-}\left(t_{0}, c_{1}, c_{2}, \varepsilon\right)\right), i=1,2 .
$$

It is easy to obtain that for each $\varepsilon \in\left[0, \varepsilon_{1}\right]$ and fixed $u_{10}, u_{20}$ the operator $J$ is equicontinuous on $S$ and maps $S$ into itself if constant $\eta_{0}$ in the theorem 1 satisfy the following condition $\eta_{0}<\frac{1}{8 l}$.

According to Schauder theorem it follows that the mapping $J$ has at least one fixed point in $S$. Let $\left(c_{1}^{*}, c_{2}^{*}\right)$ denote this fixed point. Then

$$
c_{1}^{*}, c_{2}^{*}, q_{t_{0}}^{*}=z_{t_{0}}-v^{*-}\left(t_{0}, c_{1}^{*}, c_{2}^{*}, \varepsilon\right), \quad \omega_{t_{0}}^{*}=y_{t_{0}}-w^{*-}\left(t_{0}, c_{1}^{*}, c_{2}^{*}, \varepsilon\right)
$$

satisfy the system (5.5).

The next assertion follows from theorem 1.

Theorem 2. Let the conditions I-II are valid. Then for all sufficiently small $\varepsilon$ the trivial solution of system (1.1) is stable (asymtotic stable, unstable) if and only if stable (asymtotic stable, unstable) the trivial solution of system (3.6) on center-unstable manifold.

Acknowledgement. This work was supported by the State Found of Fundamental Researches of Ukraine (grant 01.07/00047).

\section{REFERENCES}

[1] Strygin, V.V. and Sobolev, V.A.: Division Motions by Means of the Method of Integral Manifolds, Nauka, Moscow, (1987). (in Russian)

[2] Voropaeva, N.V. and Sobolev, V.A.: Constructive method of the division of nonlinear singularly perturbed differential systems, Differential Equations, 31, (4), (1995), 569-578. (in Russian)

[3] Sакамото, K.: Invariant manifolds in singular perturbation problems for ordinary differential equations, Proc. Roy. Soc. Edinburgh, 116A, (1990), 45-78.

[4] Halanay, A.: An invariant surface for some linear singularly perturbed systems with time lag, J. Diff. Eqs., 2, (1), (1966), 33-46. 
[5] Fodchuk, V.I. and Cherevko, I.M.: On the theory of the integral manifolds for singularly perturbed differential-difference equations, Ukrainski Mathematicheskii Zhurnal, 34, (6), (1982), 725-731. (in Russian).

[6] Strygin, V.V. and Fridman, E.M.: Asymptotic of integral manifolds of singularly perturbed systems of differential equations with time lag, Math. Nachr., 117, (1984), 83-109.

[7] Mitropolsky, Y.A., Fodchuk, V.I. and Klevchuk, I.I.: Integral manifolds, stability and bifurcation of solutions of singularly perturbed functional differential equations, Ukrainski Mathematicheskii Zhurnal, 38, (3), (1986), 335-340. (in Russian).

[8] Cherevko, I.M.: On integral manifolds of singularly perturbed functional differential equations, Nonlinear Oscillations, 3, (4), (2000), 562-570. (in Ukrainian).

[9] LizAnA, M.: Global analysis of the sunflower equation with small delay, Nonlinear Anal., 36, (6), (1999), 697-706.

[10] Kelley, A.: The stable, center-stable, center, center-unstable, unstable manifolds, J. Diff. Eqs., 3, (1967), 546-570.

[11] HALE, J.K.: Linear functional differential equations with constant coefficients, Contr. to Diff. Eqs., 2, (1963), 291-319.

[12] Hale, J.K.: Theory of Functional Differential Equations, Berlin, Springer-Verlag, (1977).

[13] Cherevko, I.M.: Estimate of fundamental matrix of singularly perturbed functional differential equations and its application, Differential Equations, 33, (2), (1997), 281283. (in Russian)

[14] Perestyuk, N.A. and Cherevko, I.M.: The center manifolds of singularly perturbed functional differential equations, Bulletin of the University of Kiev. Series: Physics \& Mathematics, 2, (2001), 159-169. (in Ukrainian). 